\title{
PaRoutes: a framework for benchmarking retrosynthesis route predictions
}

\author{
Samuel Genheden and Esben Bjerrum \\ Molecular AI, Discovery Sciences, R\&D, AstraZeneca Gothenburg, SE-431 83 Mölndal, Sweden \\ Corresponding author: samuel.genheden@astrazeneca.com
}

\begin{abstract}
We introduce a framework for benchmarking multi-step retrosynthesis methods, i.e. route predictions, called PaRoutes. The framework consists of two sets of 10,000 synthetic routes extracted from the patent literature, a list of stock compounds, and a curated set of reactions on which one-step retrosynthesis models can be trained. PaRoutes also comes with scripts to compute route quality and route diversity quantities that are important for comparing methods. We use the PaRoute framework to compare three methods implemented in the AiZynthFinder software: Monte Carlo tree search (MCTS), Retro*, and a depth-first proof-number search (DFPN) algorithm, all using a template-based one-step retrosynthesis model. It is found that DFPN is inferior to both MCTS and Retro* and cannot be recommended in its current implementation. MCTS and Retro* are on a par with regard to search speed and the ability to find routes in which all starting material is in stock. However, MCTS outperforms Retro* when it comes to route quality and route diversity. MCTS more easily recovers the reference routes and tends to find a diverse set of solutions for a greater portion of the targets. We encourage practitioners and developers to benchmark their algorithms using PaRoutes and we envisage that the framework will become the community standard to compare retrosynthesis route predictions. It is available at https://github.com/MolecularAI/PaRoutes
\end{abstract}

\section{Introduction}

Computer-aided synthesis planning (CASP) is a field of intense research that can provide insight and accelerate the synthesis of novel compounds, both in early discovery and late-stage development $[1,2]$. One particular area of CASP is retrosynthesis analysis in which the aim is to predict the necessary steps to synthesize a compound, i.e. a synthetic route (see Figure 1). Such methods date back to the 60 's and the early work of Corey [3], although the research has intensified in the last decade due to the increased interest in machine learning and artificial intelligence. At the heart of retrosynthesis analysis is a method that is capable of predicting disconnections on a compound and thereby producing precursors. Such methods are typically referred to as one-step retrosynthesis or single-step retrosynthesis. The precursors produced by the one-step retrosynthesis can then be further broken down recursively until a set of conditions are met. Such iterative methods are typically referred to as multi-step retrosynthesis. Typically, the stop conditions are that a precursor is found in a database of purchasable compounds, i.e. a stock, or that a maximum number of disconnections has been applied. 


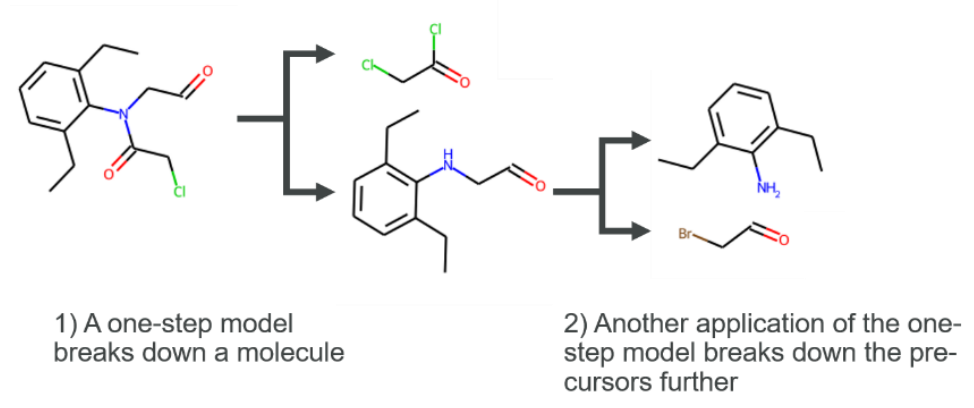

Figure 1 - The synthetic route of 2-chloro-N-(2,6-diethylphenyl)-N-(2-oxoethyl)acetamide extracted from the US03983174 patent. The figure also illustrates the difference between one-step and multi-step retrosynthesis.

One-step retrosynthesis methods have received the greatest amount of attention: there is a plethora of methods described in the literature that uses a diverse range of methods to extract synthesis rules, cheminformatic representations, neural network architectures, sampling techniques, etc. [see e.g. 4, 5, $6,7,8,9,10,11,12,13,14]$. For the practitioner that wants to use a method for predictions, or for a researcher developing a novel method, there are several comparisons for a subset of the available methods. In fact, once a novel method is developed it is customary to benchmark it against other methods using a common set of known reactions. The US patent office (USPTO) extracts provided by Lowe [15] is the de-facto standard for comparing single-step retrosynthesis method, as it is one of few open-source datasets of chemical reactions. However, there more than one curated subset of the dataset used [16, 17], which is a complication. Furthermore, there has not been a survey published showing all one-step methods side by side, so it is still difficult to understand the range of available methods.

Multi-step retrosynthesis methods, or route prediction methods, have received less attention. To the practitioner, the pool of available solutions is dominated by commercial and closed-source alternatives [2], although a few complete, open-source packages have emerged, such as the ASKCOS suite from MIT [18] and AiZynthFinder from AstraZeneca [19]. In addition, there have been a few algorithms described in the literature, which we will summarize in Section 4. What is lacking with regards to the route prediction methods is comparative studies. There is a lack of consensus on how to compare route predictions and what data one should do the comparison on. One reason for this could be that there is no public database of synthetic routes, as there is the USPTO data for single-step reactions. Another reason could be that there is typically no unique way to synthesize a compound and several alternative routes could be used that are optimal for different scenarios. With regards to the data problem, there have been a few attempts to collect a set of routes on which one can perform a comparison: Heifets and Jurisica [20] compiled a suite of routes from organic chemistry examinations that they then used to benchmark their approach. Unfortunately, the suite is extremely small, only 20 routes, and it is therefore difficult to make statistical analysis of the route predictions. Chen et al. [21] extracted routes from the USPTO dataset to train a neural network for computing the cost of synthesizing a molecule. They found all compounds in the USPTO dataset that had a route to compounds in the eMolecules database, ${ }^{1}$ and then performed further selections to arrive at a test set of size 189. Interestingly, they also used this to compare four different multi-step retrosynthesis methods (and variants thereof). They focused the comparison on computing time, the number of targets for which a route was found, and the lengths of the routes. Finally, Mo et al. [22] extracted also routes from the USPTO dataset for training their neural network for predicting the human-likeness of routes. Instead of extracting routes from the full reaction network as in [21] they extracted routes within a patent. Each patent consist of one or more reactions and a reaction network would have highly related reactions. Using a complete depth-first search of such networks for each patent, $238 \mathrm{~K}$ routes were extracted. This is an excellent number of routes for training a neural network model but is probably too excessive for benchmarking route prediction methods. However, we will build upon this methodology in Section 2 to extract routes suitable for benchmarking

\footnotetext{
${ }^{1}$ http://downloads.emolecules.com/free/
} 
multi-step retrosynthesis tools. We will then proceed to discuss and suggest metrics to compute when comparing predictions on these routes in Section 3. Finally, we will apply this framework on an illustrative comparison of three kinds of search algorithms implemented in the AiZynthFinder software in Sections 4 and 5.

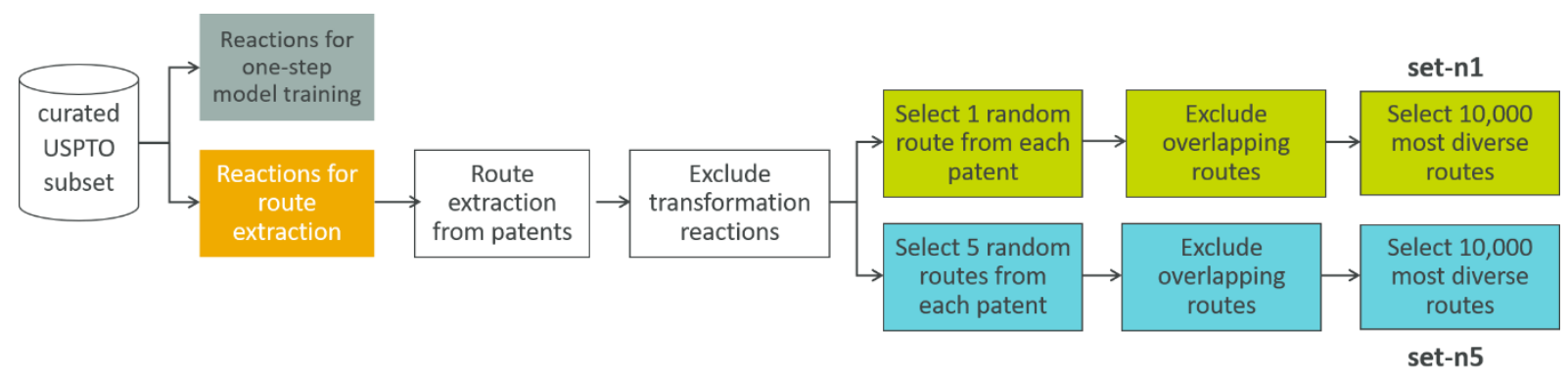

Figure 2 - Procedure to extract reference routes from a curated subset of the USPTO database. Details of the procedure is outlined in Section 2.

\section{A benchmark set for route predictions}

\subsection{Route extraction}

To extract routes, we started from the subset of the USPTO dataset prepared by Thakkar et al. [23], as this is a dataset that contains atom-mapped reactions and reaction templates, which is necessary for training a template-based one-step retrosynthesis model. We kept only reactions for which the template occurrence was four or more, i.e. all the reactions in the dataset are represented by a reaction template that occurs at least four times in the dataset. This amounts to 867,620 reactions. When extracting the routes from this dataset, we left out 99,093 reactions, three examples for each unique reaction template, to be able to have sufficient data to train a one-step model on (see below). We will now detail how routes were extracted from the 768,527 remaining reactions, a procedure summarized in Figure 2.

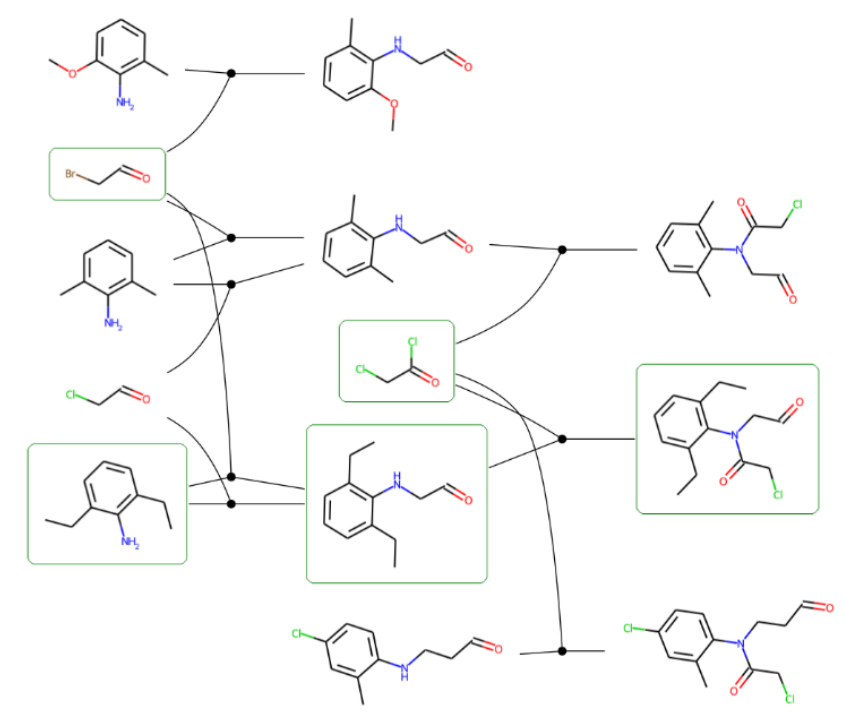

Figure 3 - Reaction network extracted from the US03983174 patent. Reactions are represented by solid circles and connects reactants to the right with the product to the left. The molecules with a green frame highlights a synthetic route for 2-chloro-N-(2,6-diethylphenyl)-N-(2-oxoethyl)acetamide and corresponds to the route in Figure 1. 
We extracted 1,046,088 routes from 80,639 patents using the method of Mo et al. [22], provided as a script $^{2}$ but with an increased timeout for finding a route from 6 to $10 \mathrm{~s}$. The script puts all the reactions from a patent in a reaction network (see an example in Figure 3), identifies molecules that only exist as a product as the starting point for synthetic routes, and then uses a depth-first search to extract the routes. After extracting the routes, we immediately discarded all routes with a single leaf, to avoid uninteresting transformation sequences, resulting in 158,698 routes. The distributions of the number of molecules, leaves, reactions, and longest linear route (LLR) are shown in Figure 4. The dataset is tilted towards short routes with few leaves. Only $4,7 \%$ of the routes are convergent; the remainder are linear. We believe the $150 \mathrm{~K}$ routes are too extensive to benchmark route predictions and it is likely that many routes from the same patent are similar. Therefore we processed the dataset further, although we acknowledge that the $150 \mathrm{~K}$ routes could be used for e.g. machine learning tasks.

We randomly selected $n$ routes from each patent and then we performed an overlap check: no route should have leaf molecules that exist in another route as intermediate precursors (non-leaves), and the target molecule should not exist as an intermediate in another route. The motivation behind this check will be clear below. We then discarded all routes with a depth of more than 10 reactions, to exclude a few really long routes that will require an extensive search. For the non-overlapping routes with a depth of at most 10 reactions, we selected the 10,000 most diverse routes: the pair-wise distance matrix was computed using the machine learning approach previously described [24] and then a greedy search with maxmin criteria was used to select the routes. We created two such sets of 10,000 routes one where $n=$ 1 and one where $n=5$, and we will refer to these two sets as set-n1 and set-n5, respectively. The distributions of the number of leaves, molecules, reactions, and longest linear route (LLR) are shown in Figure 4. For set-n1, the distributions are closer to the full set of routes: the number of molecules in the routes are typically small and the number of longer routes is quite small. On the contrary, in set-n5, the distributions are shifted to the right and there are more of the longer routes. There are $3.0 \%$ and $6.9 \%$ of convergent routes in set-n1 and set-n5, respectively.
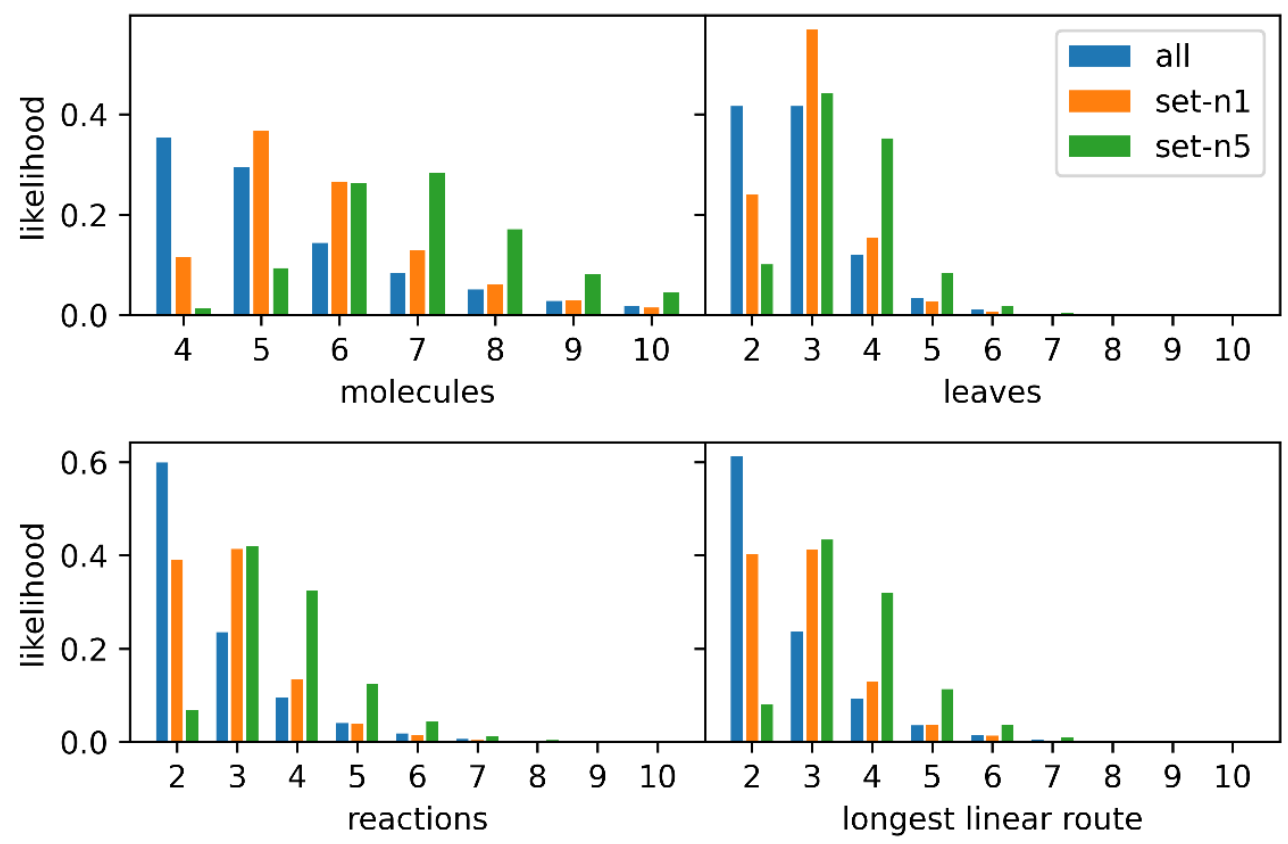

Figure 4 - The distributions of molecules, leaves, reactions and longest linear route in all routes extracted from the USPTO dataset and the two subsets created for benchmarking route predictions. The distribution has been capped at 10 and all four subplots have the same legend.

\footnotetext{
${ }^{2}$ https://github.com/moyiming1/Retrosynthesis-pathway-ranking
} 


\subsection{Stock and reaction data}

A set of routes is not sufficient to benchmark route prediction methods as there are other factors that determine the search. One such factor is the stock, the set of purchasable compounds that serve as the stop condition for the search. For creating the routes in [21] the eMolecule database was used as stop criteria, and Genheden et al. [19] created a stock from the ZINC database. Both of these alternatives are very extensive but we argue for not using such databases. Firstly, the extensiveness of the stocks could mask subtle differences between search algorithms as a route could be found by simply resorting to the stock rather than disconnecting molecules. Secondly, the quality and availability of the stock molecules are sometimes unclear, making the stop criteria arbitrary. Thirdly, databases such as eMolecules and ZINC are updated with time, making it hard to pick one representative snapshot of the database for the benchmark. We instead propose to simply use all the leaves of the 10,000 routes as stock molecules. Because we added an overlap check when extracting the routes, we can be sure that an exhaustive search would be able to find the routes without prematurely stopping because of the extensiveness of the stock.

Another factor affecting the search algorithm is of course the capability of the one-step retrosynthesis model. We have previously released a model trained on the entirety of the USPTO dataset [19, 23], but this model was trained on reactions found in the reference routes, making it biased. Using this model in the search, or indeed any one-step model trained on the reactions in the reference routes will be a mix of neural network recommendations and what amounts to literature-lookup. However, we can train a new one-step model on the data that is not found in the reference routes, and because we left out three reactions per reaction template before extracting the routes, we have sufficient data to train a templatebased one-step model. In Section 4, we will detail the training of such a model. If someone wants to extract another set of templates or train a template-free one-step model, one can always perform such modeling as well on the provided data.

\subsection{Framework summary}

To summarize the created reference set for benchmarking:

- A subset of the USPTO database with reactions that can be used to train a one-step model

- $\quad 150 \mathrm{~K}$ routes extracted from the USPTO database, which can be used for machine learning tasks

- set-n1 consisting of a diverse set of 10,000 routes which show a similar distribution in the number of molecules and reactions as the $150 \mathrm{~K}$ routes

- set-n5 consisting of a diverse set of 10,000 routes that are longer and enriched in convergent routes

- stock-n1 consisting of the 13,633 leaves molecules in set-n1 and should be used as a stock together with set-n1

- stock-n5 consisting of the 13,783 leaves molecules in set-n5 and should be used as a stock together with set-n5

The USPTO dataset, reference routes, and stocks are available as open-source together with the scripts used to create the reference routes.

We call the benchmarking set the PaRoutes (patent routes) framework. It is a framework for benchmarking route predictions because we acknowledge that not all methods are built the same. For instance, we have herein benchmarked algorithms using template-based one-step retrosynthesis methods, but there exist a plethora of alternatives. Therefore, researchers should be able to pick the parts of the framework that is applicable to their methods. If your one-step retrosynthesis model is based on templates, you can re-train it using the provided USPTO subset, which includes RDChiral-derived templates [25]. If you on the other hand want to derive templates yourself, or if you are using a template- 
free one-step model, you can use the atom-mapped reaction SMILES. Furthermore, if you have your own set of curated reactions that you want to train your one-step model on, you just have to make sure to first exclude reactions that are also in the reference routes. Because many of the existing one-step models were trained on reactions included in the reference route, we acknowledge that many of those models need to be re-trained in order to fully exploit PaRoutes.

\section{Metrics for comparing route predictions}

Whenever route prediction methods have been compared in the literature the focus has been on computational speed and the number of solved targets $[19,21,26]$, i.e. the number of targets for which at least one route is found where all the starting material is in stock. It should be noted a difference in a few seconds of search is not practically relevant, but speed is nevertheless an essential quantity for any computational method.

Although speed and number of solved targets are interesting metrics, they tell nothing about the quality of the predictions. Sometimes the quality of the routes has been quantified as the length of the routes $[21,26]$, the mean chemical complexity [27], or a metric based on the priors from the one-step model [21]. Quantifying the quality of a route by simply the route length is particularly misleading as it is very easy to envisage a shorter alternative to a route by for instance removing all protection chemistry thus rendering the route chemically infeasible. An interesting approach taken by Shibukawa et al. [27] was to sort the molecules in the routes by molecule weight and then compute the pairwise Tanimoto distances of a molecular fingerprint. Naturally, the sorting of the molecules destroys the order of the reactions in the route. We propose to use a tree edit distance (TED) method [28], which is a graph-theoretical method that recursively applies cheminformatic similarity calculations on a pair of routes to determine the similarity. By sorting the predicted routes and then computing the TED between the predictions and a reference route, one can find at what position the ground truth, i.e. the reference route is found. By doing this over all the 10,000 targets in the reference set one can compute top- $n$ accuracies, just as is standard when comparing one-step retrosynthesis methods. The top- $n$ accuracies is a metric to show how well a search method is in recovering the reference route. Naturally, other routes could be as effective or feasible as the reference route, but the only way to determine that is to perform the synthesis in the lab, which is not practically feasible for large numbers of routes. Because the routes are extracted from patents they incorporate human selection of feasible disconnections and the order of those steps. Thus, an effective search algorithm should be able to find these human-like routes.

In order to compute accuracies, we need to rank the predicted routes and we propose using the recursive route score by Badowski et al. [29] with artificial costs of leaf molecules. For intermediate molecules in a route, the cost is defined recursively as

$$
\operatorname{cost}(m)=\min _{r \in \operatorname{pred}(m)} \operatorname{cost}(r)
$$

where $\operatorname{pred}(m)$ returns the children nodes of the molecule $m$, i.e. the preceding reactions. The cost of a reaction is defined as

$$
\operatorname{cost}(r)=\varepsilon(r)+\sum_{m \in \operatorname{pred}(r)} \frac{\operatorname{cost}(m)}{\operatorname{yield}(r)}
$$

where $\varepsilon$ is a fixed cost of performing the reaction. This score is effective in ranking the route predictions based on the length and if the start material is in stock. However, the score will not differentiate by routes with similar shapes. Therefore, we can have more than one route prediction at the same rank. 
Finally, we propose a metric to quantify the diversity of the predictions. We argue that an algorithm producing more routes is not necessarily better than an algorithm producing fewer routes, because the routes can be highly similar. To compute the diversity, we compute the pairwise distance matrix of all the predicted routes using the fast machine learning method previously described [24], and then we use hierarchical clustering to group the routes. We optimize the number of clusters using the Silhouette method [30] and the optimal number of clusters is viewed as a metric of diversity.

To summarize, we suggest applying the following metrics when comparing route prediction methods on the 10,000 reference targets:

- Average search time to reach convergence in the number of solved targets

- Number of solved targets

- Top-1, top-5 and top-10 computed with the TED method

- Number of route clusters

Scripts to compute these route quality and diversity metrics are available open-source on GitHub and are considered to be part of the PaRoutes framework. We consider the calculation of timings and if targets are solved to be part of the software producing the routes. In order to compute the quality and diversity metrics, the routes need to be exported in a tree-like structure (in JSON format) with minimal features. Basically, the relationships between molecules and reactions need to be defined, molecules are defined by their SMILES string and reactions are featureless.

a) AND/OR tree

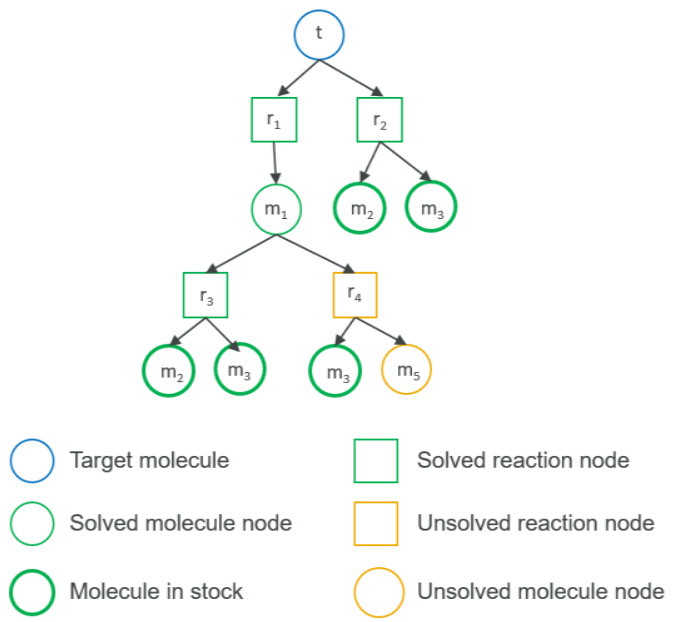

b) Super-node tree

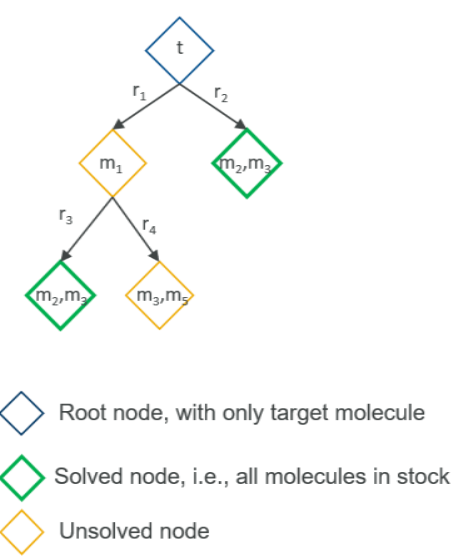

Figure 5 - Two different tree structures used in the different retrosynthesis search algorithms. The tree structures are equivalent with one target molecule $(\mathrm{t})$, pre-cursor molecules $\left(\mathrm{m}_{1}, \mathrm{~m}_{2}, \ldots, \mathrm{m}_{5}\right)$ and reactions $\left(\mathrm{r}_{1}, \mathrm{r}_{2}, \ldots, \mathrm{r}_{4}\right)$.

\section{Example application of benchmarking framework}

\subsection{Route prediction methods}

We can summarize the suggested multi-step retrosynthesis methods by dividing them into three categories: proof-number search (PNS) [31], Monte Carlo tree search (MCTS) [32], and A*-like algorithms [33]. In PNS, the retrosynthesis is carried out in a directed bipartite graph consisting of molecule and reaction nodes, i.e. an AND/OR graph (see Figure 5). The proof-number and disproof- 
number of each node, which is the number of children nodes necessary to prove and disprove a node, respectively, are used to guide the search. PNS-based retrosynthesis algorithms utilize these numbers differently and also add additional heuristics to increase the efficiency of the search [20, 26, 27]. We implemented a depth-first PNS (DFPN) algorithm in the AiZynthFinder software that combines ideas from two different algorithms [26, 27]. In MCTS, the retrosynthesis is carried out in a different type of direct graph, where each node is a super-node consisting of one or more molecules that potentially can be extended. The search is typically guided by upper-bound confidence statistics that take into account how many times a node has been visited and a reward function for terminal nodes that can take different forms $[18,34,23]$. We have previously described the MCTS implementation in the AiZynthFinder software [19], where the reward function is based on the depth of the terminal node and how many of the molecules represented by the node is in stock. Finally, several different $\mathrm{A}^{*}$-like algorithms have been described for retrosynthesis $[21,35,36]$. The Chematica program carries out the search in a supernode tree and scores the node based on customizable molecule and reaction cost functions [35]. In the Retro* algorithm, the search is carried out in an AND/OR graph and the search is guided by a molecule cost that is provided by a neural network trained on extracted routes [21]. We have implemented the Retro* algorithm in the AiZynthFinder software.

Although the algorithms that we will use the predict routes are representative of different classes of search algorithms, we acknowledge that our implementations are variants of the original implementations described in the literature. As such, the benchmarks presented herein should serve as an illustration of the PaRoutes framework and provide an insight into the capabilities of the AiZynthFinder software.

\subsection{Training a one-step model}

We selected a subset of reactions from the USPTO dataset, excluding reactions used in the reference routes, to train one-step retrosynthesis models similar to the model trained by Thakkar et al. [23]. We trained one model that was used in the set-n1 experiments and one that was used in the set-n2 experiments, although we could have trained one model on the intersection of the two datasets with some reduction in the number of reactions. The dataset consists of 878,079 and 871,001 reactions for the set-n 1 and set-n5 routes respectively, distributed over 46,092 unique reaction templates. The extracted templates were one-hot encoded as output to a neural network. The input was the extended connectivity fingerprints of the product molecules calculated by RDKit [37] using a radius of 2 and a length of 2048. The neural network was a simple feedforward network: 512 nodes with an ELU activation function, and L2 normalization, followed by a dropout later with a dropout rate of 0.4 and finally an output layer with a softmax activation function. The model was trained for 100 epochs and a batch size of 256. The Adam optimizer [38] was used with a categorical cross-entropy loss. The learning rate was initially set to 0.001 and was halved upon a plateau of the validation loss after 5 epochs. The dataset was split by $90 \% / 5 \% / 5 \%$ into training, validation, and test set, respectively.

\subsection{Details of route prediction experiments}

The target molecules of the reference routes were subjected to prediction by the AiZynthFinder software [19]. As stock we used the reference stock detailed in Section 2, i.e. all the leaves of the reference routes. We used no filter policy and the expansion policy was the one detailed above. As the search algorithm, we use an MCTS algorithm detailed previously, an implementation of the Retro* algorithm, or a depthfirst proof-number (DFPN) search. For Retro*, we used the official repository of the Retro* algorithm as a starting point, ${ }^{3}$ and re-implemented it in AiZynthFinder. An extension we implemented was continuing the search after the first solutions has been found and extraction of routes using the CompRet

\footnotetext{
${ }^{3}$ https://github.com/binghong-ml/retro_star
} 
algorithm [27]. For the DFPN, we started with the algorithm suggested by Kishimoto et al. [26]. We used a constant unity edge cost, rewrote the algorithm to be iterative rather than recursive, and implemented logic similar to [27] to continue the search after the first solution has been found. Routes were extracted using the CompRet algorithm [27]. Pseudo-code for all three search algorithms is provided in the Supporting Information. In all experiments, the search consisted of 500 iterations for MCTS and Retro*, 750 iterations for DFPN, and no time limits.

\subsection{Validation metric computations}

All routes found by either MCTS, Retro* or DFPN were scored by the route score of Badowski et al. [29]: leaf molecules in stock were assigned a cost of 1, leaf molecules not in stock were assigned a cost of 10, and all reactions were assigned a cost of $\varepsilon=1$. The top-1, top-5 and top-10 accuracies were calculated with the TED algorithm implemented in the route-distance repository. Clustering of the predicted routes was clustered using the publicly available model trained on routes for 10K ChEMBL compounds [24].

\section{Results and discussion}

The benchmark metrics listed in Section $\mathbf{3}$ are listed for all route prediction experiments in Tables $\mathbf{1}$ and 2. What follows is an analysis of those results.

Table 1 - Search performance

\begin{tabular}{|c|c|c|c|c|c|c|}
\hline $\begin{array}{l}\text { Search } \\
\text { method }\end{array}$ & Route set & $\begin{array}{l}\text { Solved } \\
\text { targets }\end{array}$ & $\begin{array}{r}\text { Search } \\
\text { time }^{\mathrm{a}}\end{array}$ & $\begin{array}{r}\begin{array}{r}\text { First solution } \\
\text { time }^{\mathrm{a}}\end{array} \\
\end{array}$ & $\begin{array}{r}\text { One-step } \\
\text { model calls }\end{array}$ & $\begin{array}{r}\text { Template } \\
\text { applications }^{\mathrm{a}}\end{array}$ \\
\hline \multirow{2}{*}{ MCTS } & set-n1 & 9714 & 303.3 & 8.6 & 3355.6 & 8658.2 \\
\hline & set-n5 & 9676 & 365.7 & 11.7 & 3615.3 & 8953.0 \\
\hline \multirow{2}{*}{ Retro* } & set-n1 & 9726 & 300.7 & 7.0 & 497.4 & 24281.1 \\
\hline & set-n5 & 9703 & 349.2 & 10.5 & 498.0 & 24322.5 \\
\hline \multirow{2}{*}{ DFPN } & set-n1 & 8475 & 347.3 & 43.0 & 404.5 & 19503.2 \\
\hline & set-n5 & 7382 & 297.9 & 53.2 & 414.5 & 19957.6 \\
\hline
\end{tabular}

\subsection{Search timings}

We will start with making an analysis of the overall search timings, and we will start with the predictions on the set-n1 routes. The retrosynthesis search was performed until 500 iterations had elapsed for MCTS and Retro*, but 750 iterations for DFPN. The reason for this is that much fewer retrosynthesis tasks, i.e. call to one-step model and template application, are done each iteration. For MCTS, the rollout implementation ensures that several calls to the one-step model and template applications are performed at each iteration. For Retro*, only a single call to the one-step model is performed each iteration. But on the other hand, one sub-tree is added to the search tree for each applicable template suggested by the one-step model, requiring several template applications. The DFPN implementation also only calls the one-step model once per iteration, but contrary to Retro* only a single sub-tree is added to the search tree, the one for the most promising template. These implementation differences are reflected in the average number of calls to the one-step model and the average number of template applications. MCTS makes on average 7 to 8 times as many calls to the one-step model as Retro* and DFPN, respectively, whereas Retro* and DFPN make 3 to 2 times as many template applications, respectively. However, more importantly, the difference in the amount of work performed in each iteration is reflected in the average search time shown in Table 1. The average search time is 303, 301, 347 s for MCTS, Retro* 
and, DFPN, respectively. Thus while using 1.5 times as many iterations, DFPN is not much slower than MCTS and Retro*, and the differences between the search methods are practically not important. Because of the relatively small amount of work done in each iteration, DFPN also requires more iterations to find solutions and the convergence in the number of solved targets is slower, as shown in Figure 6. For MCTS and Retro*, the fraction of solved target is converged at 100 iterations, and thereafter the fraction increases only very slowly. However, for DFPN it is unclear if the fraction of solved targets has converged to the same degree even after 750 iterations.

Because of the different amounts of one-step calls and template applications performed at each iteration, it is hard to benchmark the search methods based on the search time. However, when focusing on the time it takes to find a solution, DFPN is clearly outperformed by MCTS and Retro*. This can also be seen in the average time to find the first solution in Table 1, where one can observe that DFPN is approximately 6 times slower than MCTS and Retro* in finding the first solution. Analyzing the predictions on the set-n5 routes, we find that for these targets, the algorithms are generally slower finding the first solutions than searches on the set-n1, indicating that these compounds are more complex.

With respect to the comparison of MCTS and Retro*, there are some implementation details to consider. In the paper introducing Retro*, it was argued that Retro* was advantageous over MCTS because it made fewer calls to the one-step model [21]. However, in our implementation of MCTS, the template application is deferred until it is necessary, heavily reducing the number of template applications. Thus for the template-based one-step models used herein, we observe that MCTS and Retro* are approximately equally fast to reach convergence in the number of solved routes. However, it is becoming more and more popular with template-free one-step retrosynthesis models, e.g. transformerlike architectures. Such machine learning (ML) models are much slower than the simple feed-forward architecture used in the template-based model. Therefore, using a more expensive ML model or template-free methods that do not need to apply a template would tip the speed advantage to the favor of Retro*.

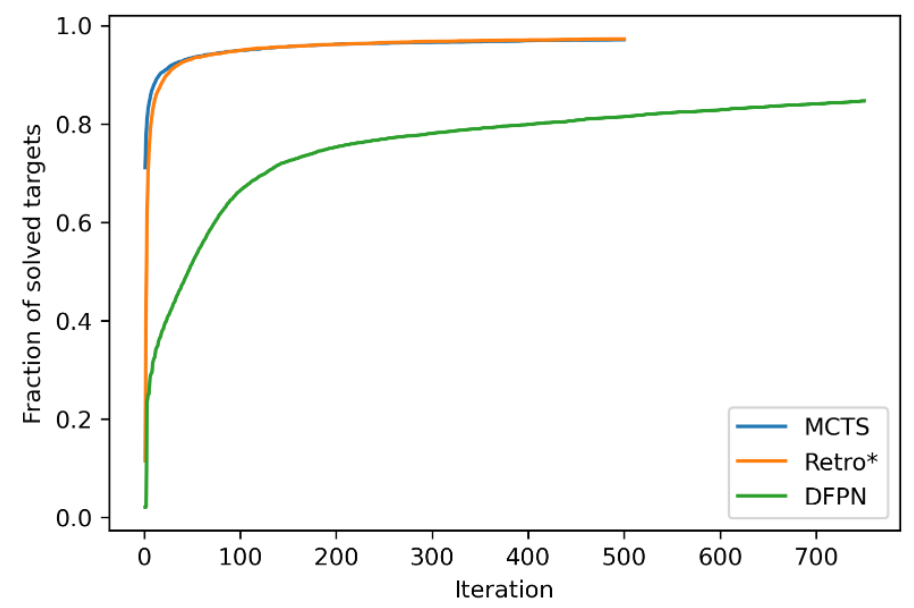

Figure 6. The fraction of solved set-n1 targets as a function of the number of elapsed iterations.

\subsection{Number of solved routes}

The goal of any retrosynthesis algorithm is to find routes where all the starting material is known, i.e., solved routes. In Table 1, we list the number of targets for which at least one solution has been found. For the set-n1 routes, both MCTS and Retro* find solutions to about $97 \%$ of the targets, and the small difference is probably not statistically significant. However, DFPN only manages to find solutions for 
$85 \%$ of the targets, a significant decrease in performance. It is unclear how many iterations are necessary to reach the performance of MCTS and Retro*, but the extra search time is most likely not warranted.

As discussed in Section 2, the distributions of the route shapes for the set-n1 follow closely the distributions for the full set of routes extracted from the patent data. However, we also extracted a set of routes that are enriched in longer and convergent routes, which presumable should be a greater challenge to the search algorithms. For all three search algorithms, we indeed observe a decrease in the number of solved targets when comparing the predictions made on the set-n 1 routes and the set-n 5 routes (see Table 1). Encouragingly, the decrease is typically rather small, between a fraction of percentage and a few percentages, for MCTS and Retro*. This shows that even though these algorithms require some more time to find a solution, they are capable of breaking down the compounds to starting material in stock. However, for DFPN, the decrease in the number of solved routes is on the order of $10 \%$, a significant amount. This shows that DFPN not only is inferior to MCTS and Retro* in finding solutions but that its capabilities worsen with compound complexity.

In SI, Table S1, we make a cross-comparison of the different search methods on the set-n1 routes to find if they are complementary or not. We find that MCTS and Retro* find solutions to the same targets, and there is a practically negligible fraction of targets for which Retro* only finds a solution. Furthermore, DFPN solves no unique targets but both MCTS and Retro* find solutions to target for which DFPN finds no solution.

Table 2 - Route quality and diversity

\begin{tabular}{|c|c|c|c|c|c|c|c|c|}
\hline $\begin{array}{l}\text { Search } \\
\text { method }\end{array}$ & $\begin{array}{l}\text { Route } \\
\text { set }\end{array}$ & top-1 & $\begin{array}{c}\text { Accurac } \\
\text { top-5 }\end{array}$ & top-10 & $\begin{array}{r}\begin{array}{r}\text { Shorter } \\
\text { route }^{\mathrm{a}}\end{array} \\
\end{array}$ & $\begin{array}{r}\text { Leaves } \\
\text { overlap }^{\text {b }}\end{array}$ & $\begin{array}{r}\text { Routes } \\
\text { extracted }^{\mathrm{c}} \\
\end{array}$ & $\begin{array}{r}\text { Number of } \\
\text { clusters }\end{array}$ \\
\hline \multirow{2}{*}{ MCTS } & set-n1 & 0.20 & 0.55 & 0.61 & 0.44 & 0.68 & 273 & 68 \\
\hline & set-n5 & 0.09 & 0.34 & 0.42 & 0.59 & 0.62 & 272 & 77 \\
\hline \multirow{2}{*}{ Retro* } & set-n1 & 0.17 & 0.48 & 0.54 & 0.44 & 0.68 & 264 & 68 \\
\hline & set-n5 & 0.08 & 0.30 & 0.38 & 0.61 & 0.63 & 149 & 39 \\
\hline \multirow{2}{*}{ DFPN } & set-n1 & 0.19 & 0.33 & 0.33 & 0.45 & 0.63 & 6 & 2 \\
\hline & set-n5 & 0.08 & 0.14 & 0.14 & 0.65 & 0.55 & 6 & 2 \\
\hline
\end{tabular}

${ }^{\mathrm{a}}$ The average number of targets for which a shorter route than the reference route is found in top- $1^{\mathrm{b}}$ The average leaves overlap in top-1. The leaves overlap is the maximum overlap between the leaves in a predicted route and the leaves in the reference route ${ }^{\mathrm{c}}$ Medians over all targets

\subsection{Route quality}

As argued in Section 3, finding routes is not a complete quality metric to assess different search methods. To be useful to a chemist, the predicted routes should be feasible, i.e. each step in the route should yield the predicted product under some reasonable conditions. We suggest computing top- $n$ accuracies, similarly to what is typically done with a one-step model, using a tree edit distance (TED) method to compute the similarity between the predicted routes and a reference route. In Table 2, we list the top-1, top-5, and top-10 accuracies. Computing higher top- $n$ accuracies are not particularly useful as there are very few lower-ranked routes because the route score used to rank the routes is rather indiscriminatory. Using MCTS on the set-n1, we arrive at a top-1 accuracy of 0.20 , which means that for roughly $1 / 5$ of targets, we find the reference ranked first. Top-10 accuracy is 0.61 , implying just for a little bit more than half of the targets, the search finds the reference ranked in the top-10. Using Retro*, we consistently achieve lower accuracies on the set-n 1 routes, with top- 1 and top- 10 of 0.17 and 0.54 , respectively, a significant drop in accuracy. DFPN is on a par with MCTS on top-1 accuracies but is worse than both 
MCTS and Retro* on top-5 and top-10 accuracies. Furthermore, for DFPN there is no difference between top-5 and top-10 accuracies, highlighting again the convergence issue with DFPN.

Table 3 - Cross comparison on ability to find the set-n1 reference routes

\begin{tabular}{ll|llll}
\hline & & \multicolumn{4}{|c}{ Found by } \\
Method 1 & Method 2 & Both & Method 1 & Method 2 & Neither \\
\hline MCTS & Retro* & 0.48 & 0.15 & 0.09 & 0.29 \\
MCTS & DFPN & 0.33 & 0.30 & 0.01 & 0.37 \\
Retro* & DFPN & 0.27 & 0.30 & 0.07 & 0.37 \\
\hline
\end{tabular}

To compare the different search algorithms further, we performed a cross-comparison: given a pair of experiments: we calculated the percentage of targets for which the reference route was identified by both methods, by just one method or by neither of the method (see Table 3). Comparing MCTS and Retro*, we see that both find the reference for $48 \%$ of the targets, and for $29 \%$ of the targets, neither method is able to find the reference route. Interestingly, the methods are not entirely complementary because only MCTS finds the reference routes for $15 \%$ of the targets, whereas only Retro* finds the reference routes for $9 \%$ of the targets. Similar observations can be made when comparing the other pair of search methods: both MCTS and Retro* outperform DFPN in uniquely finding reference routes to varying degrees. In Figure 7, we present the routes for a target for which the reference route is found by MCTS but not Retro*, and in SI we present a few similar analyses. In the routes presented in Figure 7, we can see that the top-1 routes for both MCTS and Retro* start similar and share the first three steps. However, in the reference route, the final steps are a sulfonylation followed by an $S_{N} A r$ reaction. This is also among the extracted predictions from MCTS but not for Retro*. Instead, an alternative route is predicted with the $S_{\mathrm{N}} \mathrm{Ar}$ first and the sulfonylation second, and this is the only top-1 route predicted by Retro*. This is a route also produced by MCTS, showing that this algorithm is able to extract both solutions. It is not clear why Retro* did not recover the reference route because the template corresponding to the $\mathrm{S}_{\mathrm{N}} \mathrm{Ar}$ was suggested by the one-step model as evident from the MCTS algorithm. However, something in the prioritization of nodes to expand deemed that path unpromising, continuing with the path following from the sulfonylation template. 
A)<smiles>CC1=CC=CC1</smiles><smiles></smiles>

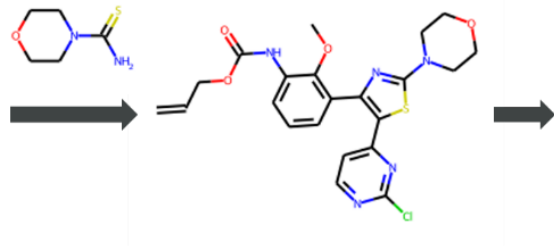

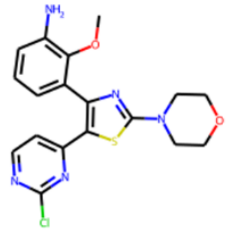

Compound A

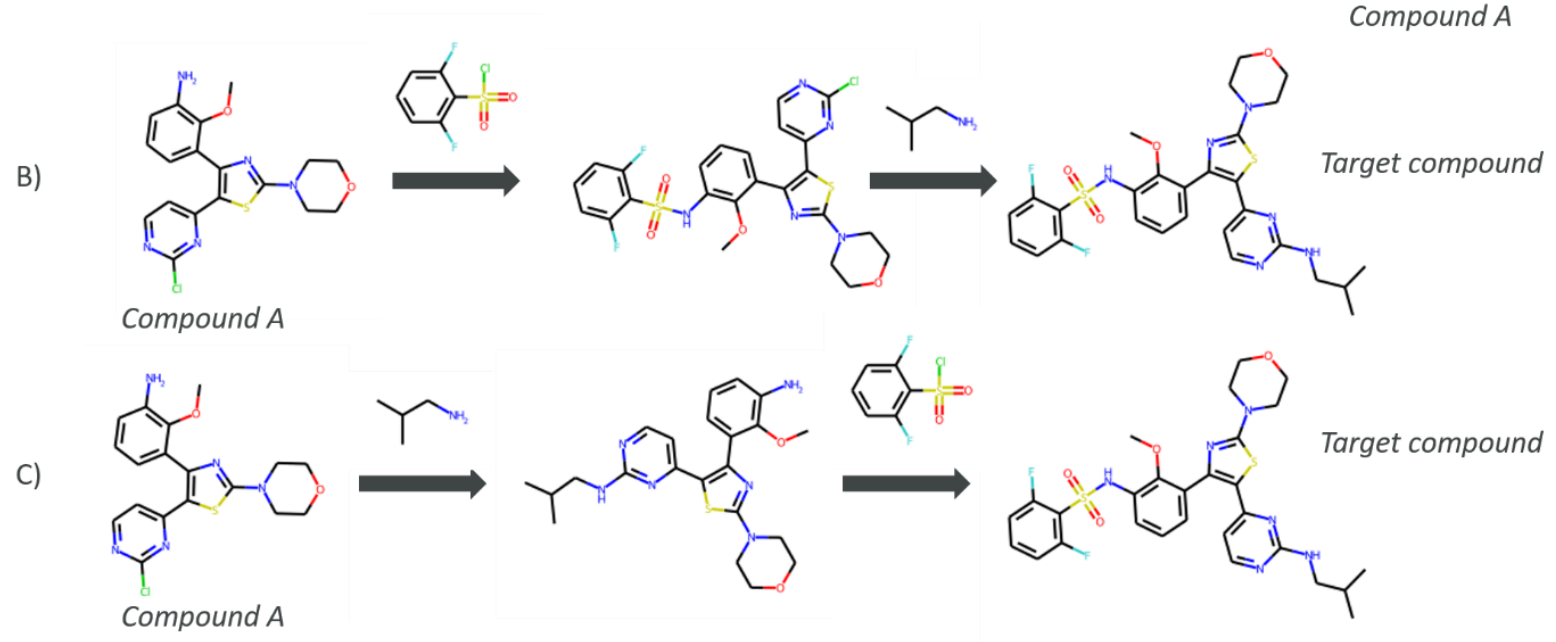

Figure 7 - Example top-ranked routes for a target where MCTS found the reference route but

Retro* was unable to. A) Route to synthesize Compound $A$ as extracted from patent

US20160060273A1, which was recovered by both MCTS and Retro*. B) Route to synthesize the target from Compound $A$ as extracted from the same patent, which was recovered by MCTS but not Retro*. C) Alternative route to synthesize the target from compound from Compound A predicted by both MCTS and Retro*.

To further investigate the rather low accuracies, we did two additional analyses that are included in Table 2. First, we calculated the fraction of targets for which a shorter route than the reference route is found in top-1. For all three methods this fraction is around 0.45 , showing that all methods were able to find alternative routes that are shorter for close to half of the targets. Second, we calculated the maximum leaves overlap between the leaves of the top- 1 ranked routes and the leaves of the reference route. An overlap of 1.0 would imply that the predicted routes have exactly the same leaves as the reference route but the reactions are not necessarily in the same order. We observe that the average leaves overlap is between 0.63 and 0.68 for the different experiments, indicating a high overlap. In fact, the leaves overlap is one for approximately $1 / 3$ of the targets in all experiments, which is significantly higher than the top1 accuracies. This shows that the different search methods were able to identify routes similar to the reference routes, but with some of the steps interchanged.

The accuracies for the predictions on the set-n 5 routes are significantly lower, with the best-observed top- 1 around 0.10 and the best top-10 around 0.42 (see Table 2). This shows more than the fraction of solved routes that set-n5 is a challenging set for any route prediction method. MCTS slightly outperforms Retro*, but it is unclear if the differences are practically relevant. There is a trend that the fraction of targets for which a shorter route than the reference route was found in top-1 is greater than for the set-n1 routes. And it is also clear that the leaves overlap in top-1 is decreased compared to the set- $\mathrm{n} 1$ routes. What this analysis show is that all of the methods are in need of improvements when it comes to finding long and convergent routes. All of the three search methods can predict shorter routes, which in Table 1 is shown as a high fraction of solved targets, but they struggle to recover the humanlike reference routes. 
a)

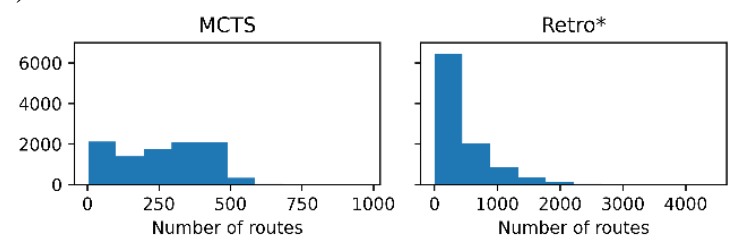

DFPN

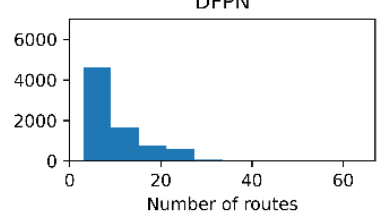

b)

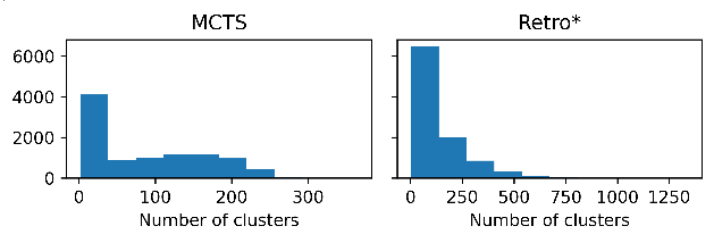

DFPN

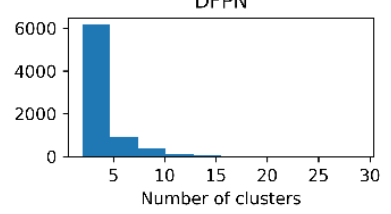

Figure 8 - the distribution of a) the number of extracted routes and b) the optimal number of clusters for each target when using the USPTO-full model on the set-n1 routes.

\subsection{Route diversity}

In Table 2, we list the median number of extracted routes and the medium number of clusters they make. For the analysis, we extracted all predicted routes that are solved, but if a target is not solved, we extract at most 25 routes. We can observe that MCTS and Retro* produce roughly equal amounts of routes, the median number of produced routes are between 149 and 273. On the other hand, DFPN produces very few routes, the median is only 6 routes. Considering that it does find solutions for close to $85 \%$ of the targets, it seems the problem with DFPN lies in its ability to find alternative solutions. This is also reflected in the lower top-5 and top-10 accuracies discussed above. The CompRet implementation of a DFPN algorithm was able to find a considerably large amount of routes for a rather small set of compounds [27], so it is possible that our implementation is inferior to CompRet in this regard. Finally, it should be noted that the distribution of the number of produced routes is heavily skewed for Retro* and DFPN, see Figure 8, whereas it is comparatively uniform for MCTS.

As argued in Section 3, it is not only the number of found routes that is important but rather the diversity of routes. To measure this, we find the optimal number of clusters formed by the predicted routes. MCTS seems to fare well in this comparison with a median of 68 for the set-n1 and 77 for the set-n5. Retro* is as successful in finding a diverse set of routes for set-n1, with a median of 68 . However, for the set-n2 the diversity is lower with a median number of clusters of 39. Because DFPN gives a low number of routes it is not surprising to see that the median number of clusters is two for both sets of routes. As seen in Figure 8 the distribution of the optimal number of clusters is also skewed. First, there is a sizable portion of the targets for which a relatively small number of clusters $(<10)$ are found and this is true for all three search methods. Then there is a roughly equal portion of targets for which the number of clusters is more than 10 for MCTS and Retro*. Finally, for Retro* we find a few targets with more than 500 clusters.

\section{Conclusion and outlook}

We have presented a framework for benchmarking multi-step retrosynthesis, i.e. route prediction, methods, and we call this framework PaRoutes (patent routes). PaRoutes consists of two sets of synthetic routes extracted from patent literature and corresponding compound stocks to be used as stop criteria for the retrosynthesis. Furthermore, it provides a curated set of reactions that can be used to train onestep retrosynthesis models. Finally, PaRoutes provides scripts to compute quality metrics: top- $n$ 
accuracies and route diversity. The entire framework is provided open-source with detailed instructions on how to use it. The framework is available at https://github.com/MolecularAI/paroutes

To illustrate the usage of PaRoutes, we have carried out route prediction experiments in order to benchmark three different search algorithms implemented in the AiZynthFinder software. We can draw some conclusions from these experiments: our implementation of DFPN is clearly inferior to MCTS and Retro*. DFPN finds fewer routes, solves fewer targets, and recovers to a lesser degree the reference routes. Further investigations are necessary to find the root cause of this, but it is clear in its current implementation, DFPN is not recommended. It should be mentioned that there are several implementations of proof-number search suggested in the literature, but all except one was designed to find a solution and then stop. The comparison of MCTS and Retro* is less clear-cut: both search algorithms solve roughly the same number of targets in roughly the same time. With respect to route quality, MCTS slightly outperforms Retro* especially for the set-n1 routes. Because both algorithms find approximately the same number of targets this shows that Retro* finds different solutions compared to MCTS. Which route would be the most chemically feasible is impossible to determine with certainty without doing experiments. However, we argue that any route significantly different from the reference route is likely inferior because the reference routes are extracted from patents, which naturally incorporate human thinking in the selected disconnections and in what order they are performed. It is worth emphasizing that all methods struggled with the set-n5 routes. Therefore, identifying and exploring human-like routes for complex targets that require longer synthetic routes seems to be an outstanding problem to solve.

PaRoute was developed and released open-source with the vision to create a community standard for benchmarking route predictions. One-step retrosynthesis is chiefly benchmarked using patent data, and we envisage that PaRoutes will become the analog for multi-step methods. We believe that the lack of comparative studies of route predictions hampers the development and is detrimental to the transparency and reproducibility of published research. Furthermore, we hope that PaRoutes can evolve with community contributions; for instance, we envisage that other quality metrics will be included in the framework when such methods are developed. By benchmarking our search algorithms we can as a community truly understand the limits of the current state-of-the-art of computer-aided retrosynthesis planning and pave the way for novel developments that have the potential to impact molecular design campaigns.

\section{Data availability}

The AiZynthFinder software is available from https://github.com/MolecularAI/aizynthfinder, and the version of the code employed for this study is 3.3.0. The PaRoutes package is available from https://github.com/MolecularAI/PaRoutes, and the version of the code employed for this study is 1.0.0. Data for the PaRoutes framework can be found at Zenodo: https://www.doi.org/10.5281/zenodo.6275421.

\section{Acknowledgements}

Jason Shields is acknowledged with proof-reading and coming with valuable suggestions on the manuscript. Alexey Voronov is acknowledged with proof-reading the manuscript.

\section{References}

1 Coley CW, Green WH, Jensen KF (2018) Machine Learning in Computer-Aided Synthesis Planning. Acc Chem Res 51:1281-1289. https://doi.org/10.1021/acs.accounts.8b00087 
2 Johansson S, Thakkar A, Kogej T, et al (2020) Al-assisted synthesis prediction. Drug Discov. Today Technol. 32:65-72 https://doi.org/10.1016/j.ddtec.2020.06.002

3 Corey EJ, Todd Wipke W (1969) Computer-assisted design of complex organic syntheses. Science 166:178192. https://doi.org/10.1126/science.166.3902.178

4 Segler MHS, Waller MP (2017) Neural-Symbolic Machine Learning for Retrosynthesis and Reaction Prediction. Chem - A Eur J 23:5966-5971. https://doi.org/10.1002/chem.201605499

5 Coley CW, Rogers L, Green WH, Jensen KF (2017) Computer-Assisted Retrosynthesis Based on Molecular Similarity. ACS Cent Sci 3:1237-1245.

https://doi.org/10.1021/ACSCENTSCI.7B00355/SUPPL_FILE/OC7B00355_SI_001.PDF

6 Liu B, Ramsundar B, Kawthekar P, et al (2017) Retrosynthetic Reaction Prediction Using Neural Sequence-to-

Sequence Models. ACS Cent Sci 3:1103-1113. https://doi.org/10.1021/acscentsci.7b00303

7 Ishida S, Terayama K, Kojima R, et al (2019) Prediction and Interpretable Visualization of Retrosynthetic

Reactions Using Graph Convolutional Networks. J Chem Inf Model 59:5026-5033.

https://doi.org/10.1021/ACS.JCIM.9B00538/SUPPL_FILE/CI9B00538_SI_002.ZIP

8 Dai H, Li C, Coley CW, et al (2019) Retrosynthesis prediction with conditional graph logic network. Adv. Neural Inf. Process. Syst. 32:8872-8882

9 Karpov P, Godin G, Tetko I V. (2019) A Transformer Model for Retrosynthesis. In: Lecture Notes in Computer Science (including subseries Lecture Notes in Artificial Intelligence and Lecture Notes in Bioinformatics).

Springer, Cham, pp 817-830

10 Sun R, Dai H, Li L, et al (2020) Energy-based View of Retrosynthesis arXiv:2007.13437

11 Fortunato ME, Coley CW, Barnes BC, Jensen KF (2020) Data Augmentation and Pretraining for Template-

Based Retrosynthetic Prediction in Computer-Aided Synthesis Planning. J Chem Inf Model 60:3398-3407.

https://doi.org/10.1021/acs.jcim.0c00403

12 Shi C, Xu M, Guo H, et al (2020) A graph to graphs framework for retrosynthesis prediction. In: 37th

International Conference on Machine Learning, ICML 2020. pp 8777-8786

13 Sacha M, Błaż M, Byrski P, et al (2021) Molecule Edit Graph Attention Network: Modeling Chemical

Reactions as Sequences of Graph Edits. J Chem Inf Model 61:3273-3284.

https://doi.org/10.1021/acs.jcim.1c00537

14 Seidl P, Renz P, Dyubankova N, et al (2021) Modern Hopfield Networks for Few-and Zero-Shot Reaction

Prediction. arXiv:2104.03279

15 D. Lowe, Chemical reactions from US patents, 1976-Sep 2016, https://figshare.com/articles/

Chemical_reactions_from_US_patents_1976-Sep2016_/5104873

16 Schneider N, Stiefl N, Landrum GA (2016) What's What: The (Nearly) Definitive Guide to Reaction Role Assignment. J Chem Inf Model 56:2336-2346.

https://doi.org/10.1021/ACS.JCIM.6B00564/SUPPL_FILE/CI6B00564_SI_001.PDF

17 Jin W, Coley CW, Barzilay R, Jaakkola T (2017) Predicting organic reaction outcomes with Weisfeiler-Lehman network. In: Advances in Neural Information Processing Systems. pp 2608-2617

18 Coley CW, Thomas DA, Lummiss JAM, et al (2019) A robotic platform for flow synthesis of organic

compounds informed by Al planning. Science 365:eaax1566 https://doi.org/10.1126/science.aax1566

19 Genheden S, Thakkar A, Chadimová V, et al (2020) AiZynthFinder: a fast, robust and flexible open-source

software for retrosynthetic planning. J Cheminform 12:70. https://doi.org/10.1186/s13321-020-00472-1

20 Heifets A, Jurisica I (2012) Construction of New Medicines via Game Proof Search In: Twenty-Sixth AAAI

Conference on Artificial Intelligence

21 Chen B, Li C, Dai H, Song L (2020) Retro*: Learning retrosynthetic planning with neural guided A* search. In:

37th International Conference on Machine Learning, ICML 2020. pp 1586-1594

22 Mo Y, Guan Y, Verma P, et al (2021) Evaluating and clustering retrosynthesis pathways with learned

strategy. Chem Sci 12:1469-1478. https://doi.org/10.1039/d0sc05078d

23 Thakkar A, Kogej T, Reymond JL, et al (2020) Datasets and their influence on the development of computer assisted synthesis planning tools in the pharmaceutical domain. Chem Sci 11:154-168.

24 Genheden S, Engkvist O, Bjerrum EJ (2021) Fast Prediction of Distances Between Synthetic Routes with Deep Learning. ChemRxiv. https://doi.org/10.26434/CHEMRXIV.14778150.V1

25 Coley CW, Green WH, Jensen KF (2019) RDChiral: An RDKit Wrapper for Handling Stereochemistry in

Retrosynthetic Template Extraction and Application. J Chem Inf Model 59:2529-2537.

https://doi.org/10.1021/acs.jcim.9b00286

26 Kishimoto A, Buesser B, Chen B, Botea Eaton A (2019) Depth-First Proof-Number Search with Heuristic Edge Cost and Application to Chemical Synthesis Planning 
27 Shibukawa R, Ishida S, Yoshizoe K, et al (2020) CompRet: A comprehensive recommendation framework for chemical synthesis planning with algorithmic enumeration. J Cheminform 12:52.

https://doi.org/10.1186/s13321-020-00452-5

28 Genheden S, Engkvist O, Bjerrum E (2021) Clustering of Synthetic Routes Using Tree Edit Distance. J Chem Inf Model 61:3899-3907. https://doi.org/10.1021/

29 Badowski T, Molga K, Grzybowski BA (2019) Selection of cost-effective yet chemically diverse pathways from the networks of computer-generated retrosynthetic plans. Chem Sci 10:4640-4651.

https://doi.org/10.1039/c8sc05611k

30 Rousseeuw PJ (1987) Silhouettes: A graphical aid to the interpretation and validation of cluster analysis. J

Comput Appl Math 20:53-65. https://doi.org/10.1016/0377-0427(87)90125-7

31 Allis LV, van der Meulen M, van den Herik HJ (1994) Proof-number search. Artif Intell 66:91-124.

https://doi.org/10.1016/0004-3702(94)90004-3

32 Browne C, Powley E, Whitehouse D, et al (2012) A Survey of Monte Carlo Tree Search Methods. IEEE Trans Comput Intell AI GAMES 4:1-43. https://doi.org/10.1109/TCIAIG.2012.2186810

33 Hart PE, Nilsson NJ, Raphael B (1968) A Formal Basis for the Heuristic Determination of Minimum Cost Paths. IEEE Trans Syst Sci Cybern 4:100-107. https://doi.org/10.1109/TSSC.1968.300136

34 Segler MHS, Preuss M, Waller MP (2018) Planning chemical syntheses with deep neural networks and symbolic Al. Nature 555:604-610. https://doi.org/10.1038/nature25978

35 Klucznik T, Mikulak-Klucznik B, McCormack MP, et al (2018) Efficient Syntheses of Diverse, Medicinally Relevant Targets Planned by Computer and Executed in the Laboratory. Chem 4:522-532.

https://doi.org/10.1016/j.chempr.2018.02.002

36 Jeong J, Lee N, Shin Y, Shin D (2021) Intelligent generation of optimal synthetic pathways based on knowledge graph inference and retrosynthetic predictions using reaction big data. J Taiwan Inst Chem Eng In press. https://doi.org/10.1016/j.jtice.2021.07.015

37 RDKit: Open-source cheminformatics, http://www.rdkit.org.

38 Kingma DP, Ba JL (2015) Adam: A method for stochastic optimization. In: 3rd International Conference on Learning Representations, ICLR 2015 - Conference Track Proceedings. International Conference on Learning Representations, ICLR 


\section{Supporting Information}

PaRoutes: a framework for benchmarking retrosynthesis route predictions

Samuel Genheden and Esben Bjerrum

Molecular AI, Discovery Sciences, R\&D, AstraZeneca Gothenburg, SE-431 83 Mölndal, Sweden

Table S1 - Cross comparison on ability to find a solution for the set-n1 routes

\begin{tabular}{ll|llll}
\hline & & \multicolumn{4}{|c}{ Found by } \\
Method 1 & Method 2 & Both & Method 1 & Method 2 & Neither \\
\hline MCTS & Retro* & 0.96 & 0.01 & 0.01 & 0.02 \\
MCTS & DFPN & 0.85 & 0.13 & 0.00 & 0.03 \\
Retro* & DFPN & 0.85 & 0.13 & 0.00 & 0.03 \\
\hline
\end{tabular}


A)

A)<smiles>OC1CCCC(C2CCC2)C1</smiles>

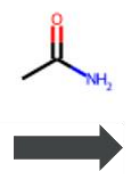<smiles>CCC(=O)c1ccc2c(c1)CC(CC(=O)C1CCC(C3CCC3)CC1)CC2</smiles><smiles>CCC(=O)c1ccc2c(c1)CCC[C]2</smiles><smiles>C=C(NC)C(=C)C1C=CC2CCCCC2=C1</smiles>

B)

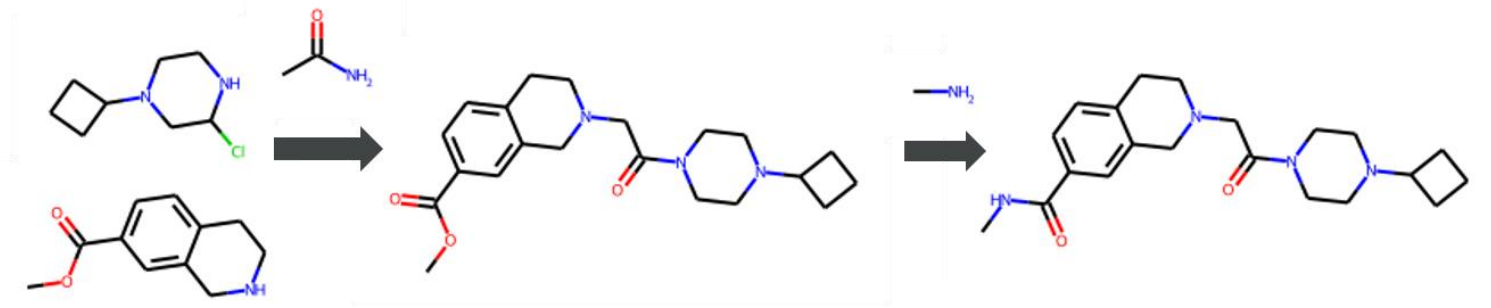

Figure S1 - Example of top-ranked route for a target where Retro* and DFPN found the reference route but MCTS was unable to. A) Route to synthesize the target as extracted from the US20070232591A1 patent, which was recovered by Retro* and DFPN. B) Alternative route to synthesize the target predicted by both MCTS. The one-step model probability and rank of the template applied on the target in route A) was $\sim 3^{-3}$ and 18 , respectively whereas the probability and rank of the template applied on the target in route B) was $\sim 2^{-2}$ and 7 , respectively. Thus Retro* and DFPN were able to utilize a template with much lower rank and could recover the reference route, whereas MCTS deemed this template to be too unlikely to continue exploring. 

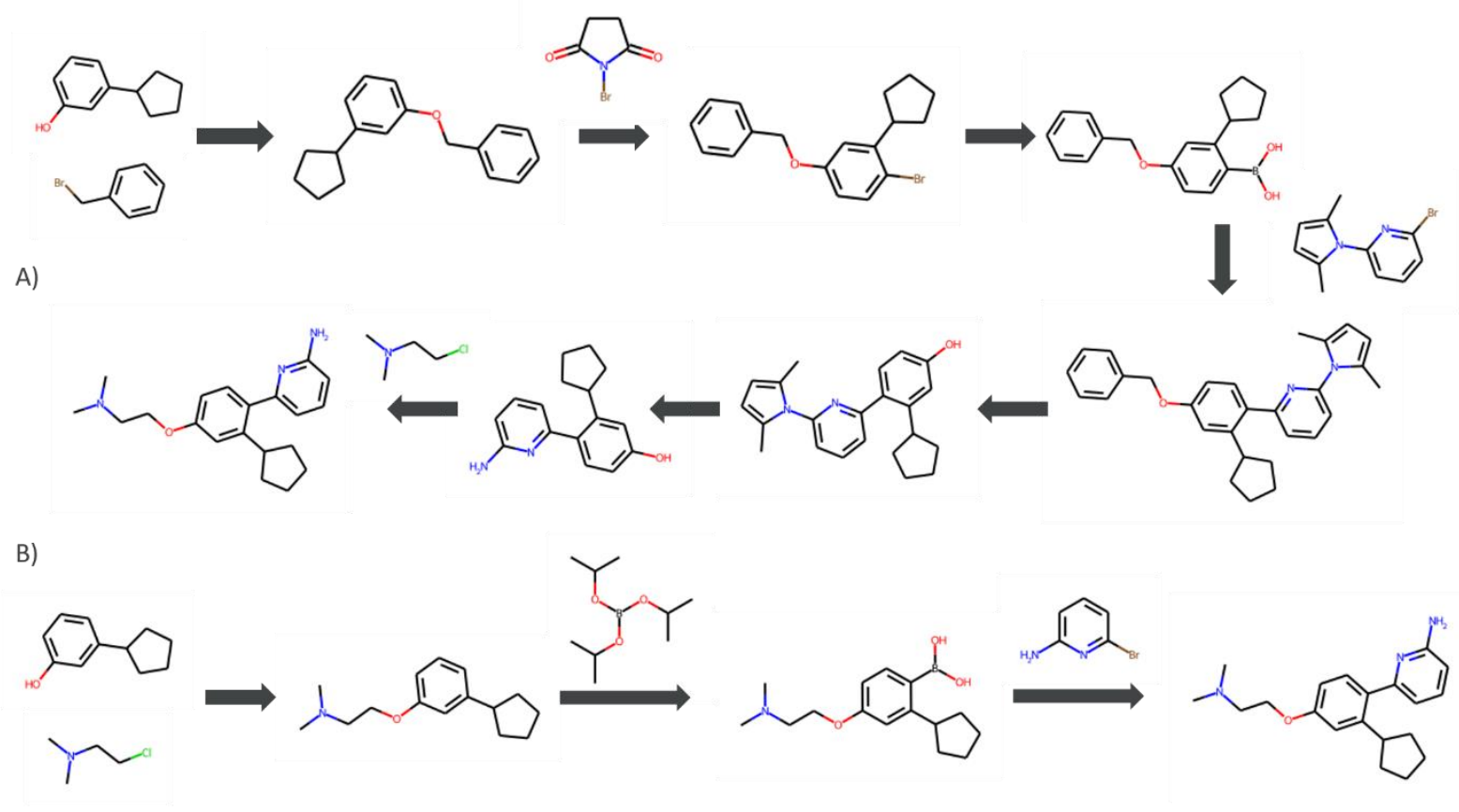

C)

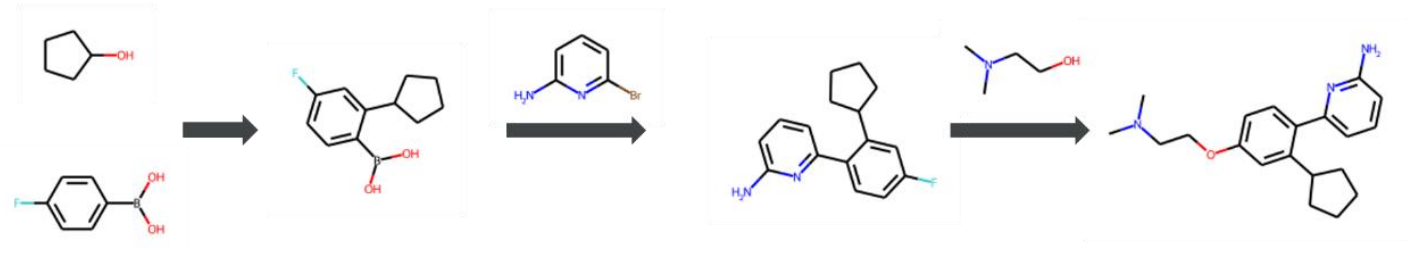

Figure S2 - Example of reference and top-ranked routes for a target where no algorithm could recover the reference route. A) The reference route extracted from patent US20030013720A1, B) topranked route from MCTS, C) top-ranked route from Retro*. Both MCTS and Retro* were able to find a shorter route to the target, but both routes will have selectivity issues. In B) there is a selectivity issue with the second step and in C) there is a selectivity issue with the first step. 
Table S2 - Overview of the search algorithms tested in this work. The boxes describe the main statements in one iteration of each of the algorithms. For clarity many of the extra checks of node states and details of various calls has been omitted.

\begin{tabular}{|c|c|c|}
\hline MCTS & Retro* & DFPN \\
\hline $\begin{array}{l}\text { \# Select leaf } \\
\text { leaf <- root } \\
\text { while leaf is expanded and leaf is not } \\
\text { terminal } \\
\text { select child with best UCB score } \\
\text { leaf <- child } \\
\text { Expand leaf with one-step model, add } \\
\text { children nodes } \\
\text { \# Rollout } \\
\text { while leaf is not terminal } \\
\text { select child with best UCB score } \\
\text { expand child with one-step model } \\
\text { leaf <- child } \\
\text { backpropagate reward of leaf }\end{array}$ & $\begin{array}{l}\text { Select leaf with minimum estimated cost from } \\
\text { all expandable leaf-nodes } \\
\text { Expand leaf with one-step model, add AND/OR } \\
\text { sub-trees } \\
\text { Update the cost of all ancestor nodes of the } \\
\text { selected leaf given the cost of the added } \\
\text { molecules }\end{array}$ & $\begin{array}{l}\text { if first iteration } \\
\text { frontier <- root } \\
\text { \# Select new frontier } \\
\text { while frontier is not expandable } \\
\text { update proof and disproof numbers of frontier } \\
\text { if frontier cannot be searched } \\
\text { frontier <- parent of frontier } \\
\text { else } \\
\quad \text { find child with minimum proof number } \\
\text { frontier <- child } \\
\text { \# Expanding molecule node } \\
\text { expand frontier, adding reaction nodes } \\
\text { update proof and disproof numbers of frontier } \\
\text { find child with minimum proof number } \\
\text { frontier <- child } \\
\text { \# Expanding reaction node } \\
\text { expand frontier adding molecule nodes } \\
\text { update proof and disproof numbers of frontier } \\
\text { find child with minimum proof number } \\
\text { frontier <- child }\end{array}$ \\
\hline
\end{tabular}


